Gran's, containing 3 per cent of salt. The presence of glucose depresses the growth of most strains.

The organisms have not been fully studied. While the finding of a halophilous organism in inland soil suggests several points of interest, the purpose of this letter is to direct attention to the probability of wide occurrence of organisms attacking agar, rather than to create new species. Such organisms have already been found in the Norwegian and West Indian seas, in Dutch soil and canal water, on Polish beetroot and Spanish raisins, and elsewhere. It seems likely that the discovery of bacteria able to attack hemicelluloses awaits but the search.

In view of the fact that such organisms have not been found here on thousands of ' count ' plates, made directly from soil suspensions, it may be of value to mention that the present organisms, as some others have been, were isolated from filter-paper strips in the course of a search for cellulose-decomposing bacteria. With these latter the agar-liquefiers were frequently associated. HugH NrCOL.

Department of Bacteriology,

Rothamsted Experimental Station, Harpenden, Herts, Dec. 9.

\section{Periodic Precipitation in the Absence of Colloids.}

Several writers 1 have recently discussed periodic precipitation in the presence of gels and other related phenomena. It is the object of this note to direct attention to one of the most important and significant papers $^{2}$ in this field that appears, however, to have been overlooked.

Morse and Pierce ${ }^{3}$ indicated in 1903 that periodic precipitation and formation of liesegang rings is not dependent upon the presence of a jelly. In his beautiful experiments described, with many photographs, in $1930,{ }^{2}$ Dr. H. W. Morse summarises his work of the intervening years and demonstrates the formation of Liesegang rings in pure aqueous inorganic substances. One of the most interesting is the series of rings of an alum, each ring consisting of a single branched crystal of the moderately soluble salt. Morse was able to explain his results without invoking anything more mysterious than the ordinary laws of diffusion and the very rapid increase in instability as supersaturated solutions rise in concentration.

Some of the first-named writers had queried the formation of rings of silver chloride in the presence of gelatine. It is interesting that this is one of the substances which Morse has photographed in periodic stratification as formed even without gelatine.

\section{Stanford University,} JaMeS W. MCBAIN.

1 Nabar and Desai, NAture, April 25, 1931, 127, 628 ; Bolam, Aug. 8, 1931, 128, 222; Bradford, Aug. 8, 1931, 128, 223; Desai, Aug. 8, $1931,128,223$; and Hedges, Sept. 5, 1931, 128, 401.

2 H. W. Morse, Jour. Phys. Chem., 34, 1554; 1930.

3 Morse and Pierce, Zeit. physik. Chem., 45, 589; 1903.

Bands in the Spectrum of Barium Hydride.

Is the last few years our knowledge of the spectra of diatomic hydrides has been greatly increased. This is especially true for the hydrides of the elements in the second column of the periodic table, the spectra of the neutral molecules $\mathrm{BeH}, \mathrm{MgH}, \mathrm{CaH}, \mathrm{ZnH}, \mathrm{CdH}$, $\mathrm{HgH}$, and of the ionised molecules $\mathrm{BeH}^{+}, \mathrm{MgH}^{+}$, $\mathrm{ZnH}^{+}, \mathrm{CdH}^{+}, \mathrm{HgH}^{+}$having been analysed. It therefore seemed worth while to complete the available information by studying the spectra of $\mathrm{SrH}$ and $\mathrm{BaH}$.

Bands probably due to $\mathrm{BaH}$ have already been reported by Eagle, ${ }^{1}$ who used an arc in a hydrogen atmosphere between electrodes containing barium chloride. The dispersion of his spectrometer was, how. ever, rather small. I have succeeded now in producing these bands with such intensity that it was possible to photograph them in the first order of a $6 \mathrm{~m}$. concave grating. The source was a vertical arc, the lower negative electrode made of nickel having the shape of a small oblong tub, containing metallic barium, while a nickel rod formed the upper positive electrode. The arc was enclosed in a cylindrical metal case con. taining hydrogen; the optimum pressure was about $4 \mathrm{~cm}$.

Four distinct band-heads were found at $\lambda \lambda 6634$, $6689,6850,6923$ A. respectively, all shaded towards the violet. The values are in agreement with the measurements of Eagle. An approximate calculation from the perfectly resolved bands furnished a moment of inertia which fits in well with the moments of inertia for elements in the second column of the periodic table, if these bands are ascribed to $\mathrm{BaH}$. The electronic transition is of the type ${ }^{2} \mathrm{II}-{ }^{2} \Sigma$ and probably is analogous to the similar transitions observed in the spectra of the other neutral molecules mentioned above. A full analysis of the spectrum will be published elsewhere.

A. SchaAfsma.

Natuurkundig Laboratorium

der Rijks-Universiteit, Groningen, Nov. 7.

Astro. Jour., 30, 231; 1909.

\section{Matter : Life : Mind.}

IN denoting matter, life, and mind as 'events' or ' grades', General Smuts struck a happy medium between the physicists' 'stages ' and the metaphysicists' 'planes'. In neither stressing nor denying a causal connexion, he succeeded in steering clear between the dangers of scientific uncertainty and meaningless profundity.

However, Prof. Boycott's letter in NATURE of Oct. 24 on this subject calls for some comment.

There will be but few dissentients in answering his query in the affirmative. But where are those 'mechanists' and 'organicists' whom Prof. Boycott saddles with assigning a quantitative relationship be. tween matter and life? Who attributes a quantitative relationship between the combined effect of lifefunctions and the heterogeneous and variable system of organised matter? I submit that in the present state of our knowledge the connexion between life and living matter is qualitative-the former being the find and determined by the complexity and organisstion rather than the mass of the latter. As to the functions by which life is perceived, we can resolve them into form, growth, orientation, and reproduction. Our physico-chemical and photoelectric experience enables us to understand and even reproduce the functions of form, growth, and direction, 1 and there is but little doubt that our research in the domain of optically active substances and radioactive isotopes will give us ere long an insight into the problem of reproduction.

As already mentioned, the functions of the living unit are related in general terms to the complexity of its organisation. In the case of viruses these functions are reduced to a minimum, with a correspondingly simplified assemblage of matter essential only to reproduction. MaURice Copisarow.

\section{Alexandra Road, Manchester, S.W., Nov. 23.}

1 Hatschek, Proc. Roy. Soe., 95 A, $303 ; 1919$. Copisarow, J. Chem. Soc., 233; 1927: and Koll. Zeits., 47, 60; 1929 : ibid., 56, 67; 1981.

$$
\text { No. } 3242 \text {, Vou. 128] }
$$

